fragments of three others which appear to be precisely similar.

Nov. 1, 1883.

\section{Supposed glacial phenomena in Boyd county, $\mathrm{Ky}$.}

A part of the work devolving upon us who have recently been tracing the southern boundary of the glaciated area in America, has been to follow up the reports of glacial phenomena south of our line.

Boyd county, Ky., having been referred to by a number of authorities as such a locality, I was naturally led to visit it a short time since; and I found, to my satisfaction, that that region was never directly glaciated.

Boyd county is in north-eastern Kentucky, bordering upon'West Virginia, and upon the remarkable bend of the Ohio River where it receives the waters of the Big Sandy. 'Through the attention of $\mathrm{Mr}$. John Campbell of Ironton, O., and Mr. J. H. Means of Ashland, Ky., I was assisted in making a pretty thorough examination of the region. Upon going back about two miles into Kentucky from the Ohio River, opposite Ironton, we find ourselves in a valley two miles wide, running parallel with the Ohio River, and two hundred and twenty feet above it. This valley extends for many miles, reaching the river towards the west at Greenup, and continuing some miles, at least, above Ashland. It is known as Flat Woods. The level is remarkably uniform; and the hills upon either side of it rise about two hundred feet, with numerous lateral openings towards the Ohio. When upon the farther side, and looking northward, one sees the rocky bluffs of the old channel rising so like those facing the river itself, that he can scarcely resist the illusion that he is in the present valley of the stream. 'The supposed glacial phenomena consist of numerous water-worn pebbles of quartz and quartzite scattered along the whole range of this old valley. Most of the pebbles are small, and perfectly rounded, though some were a foot or more in diameter; and one observed was about two feet and a half through, and only slightly worn. These pebbles are not found upon the hills back from this channel, on the Kentucky side, nor, according to Mr. Campbell, who is a most competent witness, anywhere in Lawrence county, O., back from the river. Plainly enough, they are the result of water-transportation. Whether they were deposited at the very early period when the Ohio flowed at the level of two hundred and twenty feet higher than now, and regularly occupied this old channel, or whether they were brought into place during the existence of the glacial dam which I have supposed at Cincinnati, I will not venture to say though the latter theory would seem more in accordance with the facts published by Professor White concerning the old channel followed by the Chesapeake and Ohio railroad, extending from the Kanawha River to the mouth of the Guyandotte in West Virginia. The elevation of the Kanawha-Guyandotte channel is nearly the same as that of the one I am describing, and this seems to be a prolongation of that. At any rate, the pebbles can only be indirectly referred to glacial action.

Now that attention is directed to this class of investigations, it would seem to be important for Professor Lewis to give through your columns, or somewhere else, publicity to his investigations of the facts supposed to indicate glacial action in Peunsylvania farther south than the boundary-line indicated by our investigation's two years ago.

Oberlin, Nov. 5, 1883.
Elliptic elements of comet Pons-Brooks.

While the orbit by Professor Boss, published in SCIENCE, No. 34, represents observation so well that there can be no doubt of the identity of the two comets, still it is of interest to know how closely elements derived from observations of the present comet alone agree with those of the Pons comet.

The arc of anomaly already passed over is only about twelve degrees, - a condition very unfavorable to the precise determination of elements, and inadequate to determine a reliable periodic time.

On account of this, in the solution of the equations, $\Delta e$ was considered as a known quantity, and finally an assumed value substituted for it.

I find the following corrections to Professor Boss's elliptic elements from the normal places given below:-

$$
\begin{aligned}
& \Delta \pi=-194.0^{\prime \prime} \\
& \Delta \Omega=+19.5^{\prime \prime} \\
& \Delta i=-57.5^{\prime \prime} \\
& \Delta T=-0.065235 \\
& \Delta q=+0.000716 \\
& \begin{array}{rrr} 
& 78,768 . & \Delta e \\
+\quad 289,233 . & \Delta e \\
+ & 55,256 . & \Delta e \\
+ & 108.39 & \Delta e \\
-\quad 0.04 & \Delta e
\end{array}
\end{aligned}
$$

Assuming the eccentricity to be 0.954996 , which closely approximates to the true value on the hypothesis of identity, we have for $\Delta e,-0.000274$.

The resulting corrections to the preliminary elements are, -

$$
\begin{aligned}
& \Delta \pi=-172.4^{\prime \prime} \\
& \Delta \Omega=-59.7 \\
& \Delta i=-72.6 \\
& \Delta T=-0.035537 \\
& \Delta q= \pm 0.000727 \\
& \Delta e=-0.000274
\end{aligned}
$$

\begin{tabular}{|c|c|c|c|c|c|}
\hline $\begin{array}{l}\text { Mean date, } \\
\text { Greenwich mean } \\
\text { time. }\end{array}$ & $\alpha$ & & $\delta$ & & $\begin{array}{l}\text { No. of } \\
\text { observa- } \\
\text { tions. }\end{array}$ \\
\hline 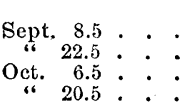 & $\begin{array}{ll}h . & m . \\
16 & 30 \\
16 & 25 \\
16 & 30 \\
16 & 45\end{array}$ & $\begin{array}{l}s . \\
38.75 \\
17.65 \\
28.52 \\
00.31\end{array}$ & $\begin{array}{ll}63^{\circ} & 49 \prime \\
60 & 45 \\
57 & 42 \\
54 & 50\end{array}$ & $\begin{array}{l}12.5^{\prime \prime} \\
52.3 \\
35.9 \\
37.4\end{array}$ & $\begin{array}{r}28 \\
16 \\
8 \\
6\end{array}$ \\
\hline
\end{tabular}

and the corrected elements are, -

$$
\begin{aligned}
& T=\text { 1884, Jan., 25.66046 } \\
& \Omega=254^{\circ} 07^{\prime} 48^{\prime \prime} \\
& \pi=\begin{array}{rrr}
93 & 18 & 50 \\
1 & =19983.0
\end{array} \\
& i=740205 \\
& \lg q=9.889708 \\
& e^{q}=0.954996
\end{aligned}
$$

After obtaining the preceding results, the equations were solved for the value of $\Delta e$, with the result $\Delta e=-0.000032$; but no use was made of this.

Normal places, 1883.0 .

These normal places are represented by the corrected elements, as follows:-

$$
\begin{array}{ccc} 
& C-0 . & \\
& \Delta a \cos \delta . & \Delta \delta . \\
\text { I. } & -0.5^{\prime \prime} & +1.3^{\prime \prime} \\
\text { II. } & -1.2 & -0.1 \\
\text { III. } & +4.4 & +0.9 \\
\text { IV. } & -0.8 & -1.2
\end{array}
$$

The last two places depend entirely upon Albany filar-micrometer observations.

In order to form some idea of the accuracy attained in modern observations of faint comets, the following table of comparisons, with the corrected elements, may be of interest. The comparisons are not very 
rigorous, and are liable to accidental errors of one or two seconds.

\begin{tabular}{|c|c|c|c|c|c|c|c|}
\hline \multicolumn{3}{|c|}{$\begin{array}{c}\text { Date, } \\
\text { Greenwich mean } \\
\text { time. }\end{array}$} & \multicolumn{3}{|c|}{ Observatory, } & $\Delta \alpha \cos \delta$ & $\Delta \delta$ \\
\hline $\begin{array}{c}\text { Sept } \\
. " \\
. " \\
. "\end{array}$ & $\begin{array}{l}3.6 \\
4.7 \\
4.7 \\
4.7 \\
5.4\end{array}$ & $\therefore:$ & $\begin{array}{l}\text { Harvard } \\
\text { Harvard } \\
\text { Harvard } \\
\text { Harvard } \\
\text { Kiel . }\end{array}$ & . & $\cdot$ & 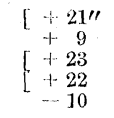 & $\left.\begin{array}{ll}- & 3 \prime \prime \\
- & 6 \\
- & 3 \\
\cdots & 2 \\
+ & 14\end{array}\right]$ \\
\hline $\begin{array}{l}" 6 \\
\because " \\
" 6 \\
" 6\end{array}$ & $\begin{array}{l}5.5 \\
5.5 \\
5.5 \\
5.6 \\
5.6\end{array}$ & $\therefore:$ & $\begin{array}{l}\text { Albany : } \\
\text { Harvard : } \\
\text { Wien : } \\
\text { Albany : } \\
\text { Cincinnati: }\end{array}$ & . & : & $\begin{array}{r}3 \\
+12 \\
-\quad 4 \\
-3 \\
0\end{array}$ & $\begin{array}{l}-7 \\
+4] \\
+\quad 1 \\
+11 \\
+12\end{array}$ \\
\hline $\begin{array}{l}66 \\
6 " \\
66\end{array}$ & $\begin{array}{l}5.6 \\
6.4 \\
6.4 \\
6.5 \\
6.6\end{array}$ & $\therefore:$ & $\begin{array}{l}\text { Jeiden . } \\
\text { Königsberg } \\
\text { Dun Kicht . } \\
\text { Harvard : } \\
\text { Albany. }\end{array}$ & : & . & $\begin{array}{r}+2 \\
+12 \\
{\left[\begin{array}{c}2 \\
+12 \\
-3\end{array}\right.}\end{array}$ & $\begin{array}{l}+10 \\
+26] \\
+\quad 3 \\
+\quad 5 \\
+\quad 2\end{array}$ \\
\hline $\begin{array}{l}4 \\
6, \\
6, \\
64\end{array}$ & $\begin{array}{l}6.6 \\
6.6 \\
6.6 \\
6.8 \\
7.3\end{array}$ & $\therefore:$ & $\begin{array}{l}\text { Albany } \\
\text { Harvard } \\
\text { Cincinnati: } \\
\text { Harvard : } \\
\text { Wien : }\end{array}$ & 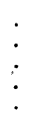 & . & $\begin{array}{r}\cdots 5 \\
-\quad 1 \\
+\quad 3 \\
+22 \\
+\quad 5\end{array}$ & $\left.\begin{array}{r}+10 \\
+10 \\
+\quad 3 \\
+\quad 0 \\
+\quad 4\end{array}\right]$ \\
\hline $\begin{array}{l}46 \\
4 \\
46 \\
46\end{array}$ & $\begin{array}{l}7.4 \\
7.5 \\
8.4 \\
8.4 \\
9.3\end{array}$ & $\therefore:$ & $\begin{array}{l}\text { Kiel . } \\
\text { Harvard } \\
\text { Leiden } \\
\text { Dun Echt } \\
\text { Pulkowa }\end{array}$ & : & $\dot{ }$ & 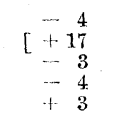 & $\begin{array}{r}+\quad 1 \\
+\quad 10 \\
-\quad 3 \\
-\quad 9 \\
-0\end{array}$ \\
\hline $\begin{array}{l}66 \\
66 \\
66 \\
66\end{array}$ & $\begin{array}{r}9.4 \\
9.5 \\
9.6 \\
9.6 \\
10.4\end{array}$ & $\therefore:$ & $\begin{array}{l}\text { Kiel. . } \\
\text { Strasburg } \\
\text { Albany : } \\
\text { Harvard : } \\
\text { Pulkowa }\end{array}$ & . & : & $\begin{array}{ll}- & 2 \\
- & 1 \\
- & .1 \\
+ & 2 \\
+ & 4\end{array}$ & $\begin{array}{l}+\quad 2 \\
+\quad 3 \\
-2 \\
-\quad 15 \\
+\quad 2\end{array}$ \\
\hline $\begin{array}{l}.6 \\
66 \\
66 \\
66\end{array}$ & $\begin{array}{l}10.5 \\
10.5 \\
10.5 \\
10.8 \\
11.5\end{array}$ & $: \vdots$ & $\begin{array}{l}\text { Kiel . } \\
\text { Dun Echt : } \\
\text { Strasburg. } \\
\text { Cincinnati: } \\
\text { Kiel . . }\end{array}$ & $\cdot$ & $\cdot$ & $\begin{array}{l}-1 \\
+\quad 1 \\
+\quad 2 \\
+\quad 7 \\
-\quad 9\end{array}$ & $\begin{array}{r}+\quad 6 \\
+\quad 1 \\
+\quad 0 \\
+\quad 5 \\
+\quad 5\end{array}$ \\
\hline $\begin{array}{l}36 \\
86 \\
46 \\
16\end{array}$ & $\begin{array}{l}11.6 \\
17.3 \\
18.5 \\
19.3 \\
19.4\end{array}$ & $\therefore:$ & $\begin{array}{l}\text { Dun Echt : } \\
\text { Pulkowa } \\
\text { Albany : } \\
\text { Kiel : } \\
\text { Strasburg : }\end{array}$ & 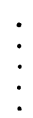 & : & $\begin{array}{r}-5 \\
+\quad 6 \\
-1 \\
-\quad 0 \\
-\quad 3\end{array}$ & $\begin{array}{l}+\quad 5 \\
+\quad 3 \\
+\quad 7 \\
-\quad 1 \\
-\quad 4\end{array}$ \\
\hline $\begin{array}{l}\text { "“ } \\
" 6 \\
4,\end{array}$ & $\begin{array}{l}21.4 \\
21.6 \\
21.6 \\
22.3 \\
23.3\end{array}$ & $\therefore:$ & $\begin{array}{l}\text { Strasburg. } \\
\text { Albany. } \\
\text { Albany : } \\
\text { Königsberg } \\
\text { Wien . }\end{array}$ & 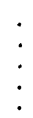 & : & 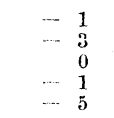 & $\begin{array}{ll}- & 5 \\
+ & 1 \\
\cdots & 7 \\
+\quad 6 \\
-\quad 2\end{array}$ \\
\hline $\begin{array}{l}.4 \\
4 \\
46 \\
s,\end{array}$ & $\begin{array}{l}23.3 \\
25.4 \\
25.6 \\
25.6 \\
26.6\end{array}$ & $\therefore:$ & $\begin{array}{l}\text { Kiel . : } \\
\text { Leiden : } \\
\text { Albany : } \\
\text { Albany : } \\
\text { Albany : }\end{array}$ & . & . & $\begin{array}{r}-\quad 6 \\
-\quad 3 \\
+11 \\
+\quad 7 \\
-1\end{array}$ & $\begin{array}{l}-\quad 2 \\
+\quad 6 \\
+6 \\
+\quad 5 \\
-7\end{array}$ \\
\hline $\begin{array}{c}", \\
\text { Oct. } \\
",\end{array}$ & $\begin{array}{r}26.6 \\
26.7 \\
3.6 \\
4.6 \\
4.6\end{array}$ & $\because:$ & $\begin{array}{l}\text { Cincinnati : } \\
\text { Albany : } \\
\text { Albany : } \\
\text { Albany: } \\
\text { Albany: }\end{array}$ & . & . & $\begin{array}{r}79 \\
+\quad 3 \\
+\quad 5 \\
+\quad 3 \\
+\quad 6\end{array}$ & $\begin{array}{r}+\quad 9 \\
+\quad 3 \\
+\quad 2 \\
+\quad 3 \\
+0\end{array}$ \\
\hline $\begin{array}{l}\$ 4 \\
" 6 \\
" 6 \\
" 6\end{array}$ & $\begin{array}{l}4.6 \\
5.5 \\
7.6 \\
7.6 \\
9.5\end{array}$ & $\therefore:$ & $\begin{array}{l}\text { Albany : } \\
\text { Albany : } \\
\text { Albany : } \\
\text { Albany: } \\
\text { Albany: }\end{array}$ & : & . & $\begin{array}{l}+3 \\
+\quad 1 \\
+\quad 6 \\
+\quad 3 \\
+\quad 8\end{array}$ & $\begin{array}{r}+\quad 2 \\
+\quad 4 \\
+\quad 2 \\
+\quad 2 \\
+\quad 5\end{array}$ \\
\hline $\begin{array}{l}.4 \\
64 \\
6, \\
66\end{array}$ & $\begin{array}{l}16.5 \\
17.5 \\
18.5 \\
21.5 \\
24.6\end{array}$ & : & $\begin{array}{l}\text { Albany : } \\
\text { Albany : } \\
\text { Albany: } \\
\text { Albany : } \\
\text { Albany: }\end{array}$ & . & ${ }^{\circ}$ & $\begin{array}{r}0 \\
+\quad 2 \\
+\quad 6 \\
+\quad 5 \\
-\quad 4\end{array}$ & $\begin{array}{r}-\quad 9 \\
+\quad 1 \\
+\quad 0 \\
+\quad 5\end{array}$ \\
\hline “ & 25.5 & . & Albany. & & • & -3 & $\cdots 5$ \\
\hline
\end{tabular}

The observations enclosed in brackets were not used as exhibiting large systematic or accidental errors.

A few observations were made with ring-micrometers, but it is not possible to determine how many.
At Albany the ring was used until Sept. 21, afterwards the filar micrometer.

The following table shows the constant difference for each observer when there are three or more observations given, and includes nothing later than Sept. 26:-

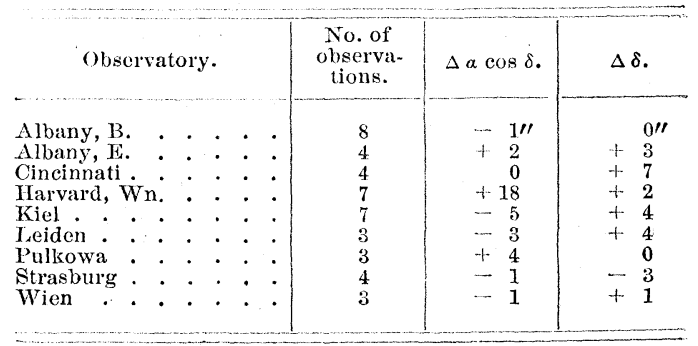

These constant errors, though founded on rather slender material, probably represent fairly what is to be expected from modern observations of comets.

Following are the heliocentric co-ordinates:-

$$
\begin{aligned}
& x=r(9.580346) \sin \left(153^{\circ} 14^{\prime} 15.1^{\prime \prime}+v\right) \\
& y=r(9.996200) \sin (8204 \quad 40.0+v) \\
& z=r(9.970401) \sin \left(\begin{array}{lll}
174 & 59 & 17.4+v)
\end{array}\right.
\end{aligned}
$$

Dudley observatory, Albany, N.Y.,

H. V. EGBERT. Nov. $6,1.883$.

\section{Rapid geological changes in Alaska.}

Mr. Dall kindly calls my attention to an error in the note of my remarks, given in SCIENCE of Oct. 19. Hood's Bay is nearly a degree south of the locality of the submerged forest described. Looking at my diary, I find the entry 'Hoona,' which is, I believe, synonymous with 'Bartlett Bay' of some charts. While making my verbal remarks at the academy, I mistook my pencilling of 'Hoona' for 'Hood.' 'The exact location of the forest is latitude $58^{\circ} 27^{\prime}$, longitude $135^{\circ} 40^{\prime}$. I am very much pleased to find from Mr. Dall's letter that my view of the modern changes, drawn from botanical facts chiefly, derives support from some geographical evidence within his reach.

Thomas Meehan.

\section{The mechanism of direction.}

I read with interest Professor Newcomb's article on the sense of direction (Scrence, Oct. 26). Professor Newcomb says nothing about the behavior of the subjective co-ordinates under a slight change of angle. $\mathrm{My}$ experience in this respect $I$ give below, and $I$ have reason to believe the experience to be quite general.

The street A B turns into B C. Walking from A to $B$, my co-ordinates begin to change when about a hundred yards from $B$. By the time I get to $B$, or rather just after $B$, they have changed by the angle $A$ B C, no matter how large or how small A B C is. The same takes place in going from $\mathrm{C}$ to $B$ to $A$. While close to $B$ on either side, I can by an effort.imagine myself under the old co-ordinates; but the new ones are much more natural. In the dark, I think the turn is not seen so far ahead, and the change takes less time. If I go from A to B, with my eyes turned towards $A, I$ have a different experience. I have never tried it by walking backwards;

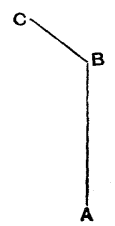
but I have observed my sensations while riding on the back platform of a street-car. As the car turns at $B$ towards $C$, and I am looking towards A, my co- 